\title{
One-pot Synthesis of 3D Hierarchical Flower-like Titanium Dioxide Microspheres with Enhanced Photocatalytic Performance
}

\author{
YANG Yu ${ }^{1, a}, X U$ Wenyan ${ }^{1, b}$, Ding Zhenbo ${ }^{1, c}$ and WANG Lei ${ }^{1, d *}$ \\ ${ }^{1}$ Key Laboratory of Eco-chemical Engineering, Ministry of Education, \\ Inorganic Synthesis and Applied Chemistry, College of Chemistry and Molecular Engineering, \\ Qingdao University of Science and Technology, Qingdao 266042, P. R. China \\ ayangyu9039@163.com, ${ }^{\mathrm{b}}$ anneyan@163.com, ${ }^{\mathrm{c}}$ ding@qust.edu.cn, ${ }^{\mathrm{d} i n o r c h e m w l @ 163 . c o m}$
}

\begin{abstract}
Keywords: $\mathrm{TiO}_{2}$; Flower-like; Hierarchical; Solvothermal; Photocatalysis
Abstract Flower-like 3D $\mathrm{TiO}_{2}$ microspheres with hierarchical structure are fabricated via a facile solvothermal approach in a solution of $\mathrm{N}, \mathrm{N}$-dimethylformamide (DMF) with the addition of diethanolamine (DEA). The microspheres with the size of 200-300 nm are composed of $\mathrm{TiO}_{2}$ nanosheets. The influence of reaction time has been studied in detail. The possible formation process of the hierarchical $\mathrm{TiO}_{2}$ microstructures is discussed on the basis of time-dependent experiments. When used as photocatalyst, as-prepared $\mathrm{TiO}_{2}$ sample exhibits highly improved activity.
\end{abstract}

\section{Introduction}

Titanium dioxide $\left(\mathrm{TiO}_{2}\right)$ has been the focus of considerable attention in recent years owing to its excellent properties in a variety of fields, such as photocatalysis, dye-sensitized solar cells and electrochemical energy storage [1-9]. Of the many different applications, $\mathrm{TiO}_{2}$ as photocatalyst has been extensively and intensively studied owing to its chemical stability, long durability, and nontoxicity. It is well known that the particle size, specific surface area, crystallinity, and morphology of $\mathrm{TiO}_{2}$ has significant influence on photocatalytic activity of $\mathrm{TiO}_{2}$ [10]. A zero-dimensional $\mathrm{TiO}_{2}$ sphere has a higher rate of photocatalytic decomposition because of its high specific surface area[11]. The two-dimensional structure of $\mathrm{TiO}_{2}$ nanosheet possesses high smoothness and adhesion, therefore extremely beneficial for photocatalytic reactions[12, 13]. Starting from the zero-dimensional nanoparticle, the hierarchical growing patterns can be further expanded [14]. Self-assembly of highly active anatase $\mathrm{TiO}_{2}$ nanoblocks to large-surface-area hierarchical microspheres has developed due to their novel architecture and their resulting fascinating properties [15-17].

The hierarchical structures of $\mathrm{TiO}_{2}$ with controllable sizes and morphologies have also become an interesting focus because of their potential applications in catalysis, photovoltaics, sensing, energy storage and environmental fields[9, 18-24]. The synthesis strategy of fabricating hierarchical architecture includes the template method and the template-free method[9]. Although the template technique is effective to direct the formation of hierarchical structure, the template will be removed by calcination or dissolution in the multi-step process. But the template-free method is a simple and environment-friendly process. Herein, we report a facile solvothermal approach of $\mathrm{TiO}_{2}$ microspheres with three-dimensional (3D) hierarchical structures, based on an Ostwald ripening mechanism. To our knowledge, there has few report on $\mathrm{TiO}_{2}$ microspheres prepared by a simple one-pot solvothermal process in a solution of $\mathrm{N}$-dimethylformamide (DMF) with the addition of diethanolamine (DEA)[25]. The possible formation process of the $3 \mathrm{D}$ hierarchical $\mathrm{TiO}_{2}$ microspheres was discussed on the basis of time-dependent experiments. The influences of reaction time, amount of DEA and reaction temperature on the morphologies and structures of $\mathrm{TiO}_{2}$ have been studied in detail. As photocatalyst, as-prepared 3D hierarchical $\mathrm{TiO}_{2}$ microspheres exhibit excellent reactivity. The fabrication of hierarchical nanostructures represents another strategy for improving photocatalytic activity of $\mathrm{TiO}_{2}$ materials by light harvesting enhanced. 


\section{Experimental}

Synthesis of 3D hierarchical $\mathbf{T i O}_{2}$ microspheres. All chemicals were analytical reagent grade and used without further purification. In a typical experiment, $1 \mathrm{~mL}$ titanium (IV) isopropoxide (TTIP) was dissolved in a mixed solution of $\mathrm{N}$-dimethylformamide (DMF) and diethanolamine (DEA) with different molar ratios under stirring, to obtain a homogeneous solution. The solution was then transferred to a $60 \mathrm{~mL}$ Teflon-lined autoclave and heated in an electric oven at $200{ }^{\circ} \mathrm{C}$ for $24 \mathrm{~h}$, and then cooled at ambient temperature naturally. The obtained product was separated by centrifugation, washed by distilled water and ethanol several times, and finally dried under vacuum at $60{ }^{\circ} \mathrm{C}$ overnight. The $\mathrm{TiO}_{2}$ microspheres were calcined at $400{ }^{\circ} \mathrm{C}$ for $2 \mathrm{~h}$ with a heating rate of $1{ }^{\circ} \mathrm{C} \mathrm{min}{ }^{-1}$.

Characterization. The crystal structure of the resulting products was examined using a Rigaku D/Max 2200-PC diffractometer (XRD) with a graphite monochromator and $\mathrm{Cu} K \alpha$ radiation $(\lambda=$ $0.15418 \mathrm{~nm}$ ) while the voltage and electric current were held at $40 \mathrm{kV}$ and $20 \mathrm{~mA}\left(2 \theta=10-70^{\circ}\right)$, respectively. The morphology and microstructure observation was performed on a JEOL JSM-7500F scanning electron microscope (SEM) and high-resolution TEM (HR-TEM, GEOL10) with an accelerating voltage of $200 \mathrm{kV}$.

Photocatalytic properties. In order to minimize the effect of scattered light by the reaction solution, the photoreactor was designed with cylindrical quartz cell configuration and an internal light source was surrounded by quartz jacket, so that the catalysts and aqueous dyes surrounded the light source completely.

The catalytic activities of the hierarchical $\mathrm{TiO}_{2}$ microspheres were characterized by the degradation of methyl orange (MO) under UV irradiation. A general photocatalytic procedure was carried out as follow. $0.005 \mathrm{~g}$ as-prepared photocatalyst was added to $10 \mathrm{~mL} \mathrm{MO}$ solution with a concentration of $5 \times 10^{-4} \mathrm{~mol} \mathrm{~L}^{-1}$. The solution was stirred in the dark for 2 hours to establish adsorption-desorption equilibrium between the dye molecules and the $\mathrm{TiO}_{2}$ catalyst. The measure was tested after the intensity of the lamp became stable. The solution was vigorously stirred during the process. All degradation experiments were carried out at $20 \pm 2{ }^{\circ} \mathrm{C}$ with the photoreactor open to air. The decrease of dyes concentration was analyzed by UV-Vis spectroscopy. At given intervals of illumination, a sample of reaction solution was taken out and then centrifuged to remove the photocatalyst particles. The upper solution was analyzed by UV-Vis spectroscopy. The light source was a $500 \mathrm{~W}$ high-pressure mercury lamp (Xu Jiang Nanjing).

\section{Results and discussion}

Structural and Morphological Characteristics. X-ray diffraction (XRD) analysis is performed to investigate the crystal phase of products prepared by solvothermal synthesis (curve I) and those being further calcination (curve II), as shown in Fig. 1. Obviously, the sample without calcination is amorphous. After the thermal process at $400{ }^{\circ} \mathrm{C}$, the product has uniform anatase structure with its characteristic diffraction peaks of $2 \theta$ values at 25.3 (101), 37.8 (004), 48.0 (200), 53.9 (105), 55.1 (211) and 62.7 (204) (JCPDS card no. 21-1272).

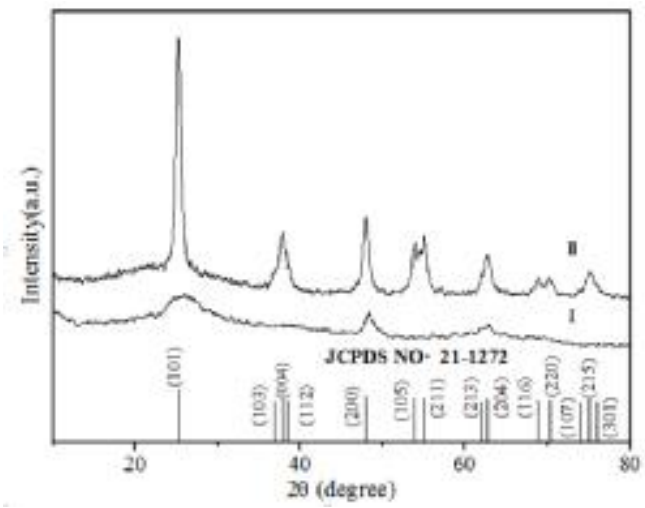

Figure 1 XRD patterns of the $\mathrm{TiO}_{2}$ sample synthesized by solvothermal treatmentat $200{ }^{\circ} \mathrm{C}$ for $24 \mathrm{~h}$, before (I) and after(II) calcination for $2 \mathrm{~h}$ under air at $400{ }^{\circ} \mathrm{C}$ 
The detailed morphology and microstructure of the product after annealing at $400{ }^{\circ} \mathrm{C}$ are characterized by FE-SEM, TEM, and HRTEM, as shown in Fig. 2a-d. From these micrographs, it is observed that the $\mathrm{TiO}_{2}$ samples are flower-like microspheres with the diameter of about 200-300 nm and these microstructures are comprised of numerous nanosheets with the thickness of about 5-10 nm. The lattice fringe with a width of $0.35 \mathrm{~nm}$ corresponds to the (101) plane of anatase $\mathrm{TiO}_{2}$. SAED pattern of $\mathrm{TiO}_{2}$ microsphere (Fig. 2e) indicates the polycrystalline nature of the sample and each of the diffraction rings can be readily indexed to anatase $\mathrm{TiO}_{2}$, which is consistent with the XRD result.

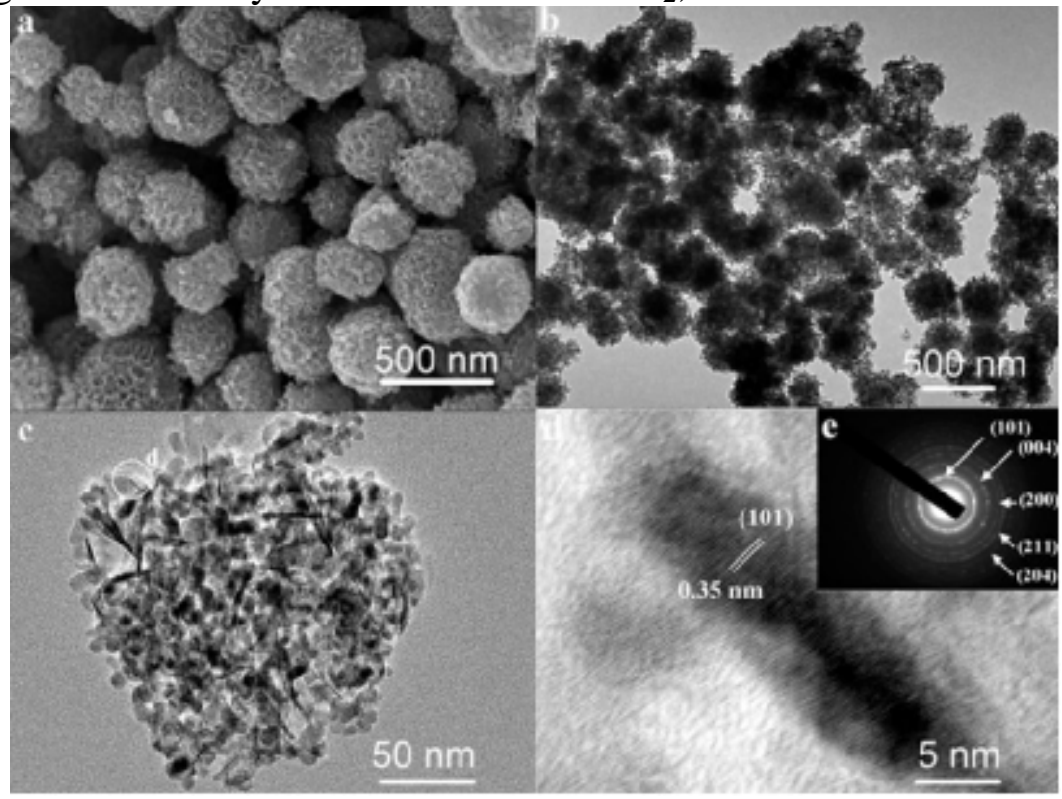

Figure 2 (a) FE-SEM micrograph, (b) TEM micrograph (c) high magnification TEM micrograph,

(d) HR-TEM micrograph of the area marked as $d$ in (c), and (e) SAED pattern of the

$\mathrm{TiO}_{2}$ sample synthesized by solvothermal treatment after calcination for $2 \mathrm{~h}$ at $400{ }^{\circ} \mathrm{C}$

In order to further explore the formation mechanism and morphological evolution of the 3D flower-like hierarchical $\mathrm{TiO}_{2}$ microspheres, time-dependent experiments are carried out and the FE-SEM results are shown in Fig. 3. The results indicate that there is no product when the reaction time is less than $6 \mathrm{~h}$. At the early reaction stage, plenty of irregular spheres generate and gather together after solvothermal treatment for $6 \mathrm{~h}$, as shown in Fig. 3a. From the amplification FE-image of a part of the sphere (Fig. 3b), the surface of the sphere is composed of slim particles. With increasing the time up to $12 \mathrm{~h}$, nanosheets occur and the agglomeration of the spheres gets obvious improvement (Fig. 3c). According to the result of Fig.3d, there is not a close packing of the sheets. After $18 \mathrm{~h}$ of solvothermal reaction, the sample is mainly made up of two-hemisphere-like microspheres and the sphere is composed of nanosheets completely, as shown in Fig. 3e and 3f. With reaction time lasting $24 \mathrm{~h}$, these two-hemisphere-like microspheres continue to grow and separate into two monodispersed microspheres with an average diameter of about $300 \mathrm{~nm}$ (Fig. $3 \mathrm{~g}$ and $3 \mathrm{~h}$ ).

On the basis of the analysis of products in different periods, the formation process of 3D flower-like hiearchical $\mathrm{TiO}_{2}$ microspheres isproposed as Scheme 1. The formation of the $\mathrm{TiO}_{2}$ microspheres may be attributed to DEA. DEA possesses two hydroxyl functional groups, which can provide coordinate ability and selectively bind to specific crystallographic facets, and can react with the Ti4+ ions to form hydroxyl complexes. These resulting less reactive complexes inhibite therapid hydrolysis of $\mathrm{Ti}^{4+}$ and promote the formation of nanoscaled particles. Then these particles self-assemble and produce sphere-like aggregation in order to reduce Gibbs free energy. At high temperature and pressure, DMF is decomposed to $\mathrm{OH}^{-}$that not only increases alkalinity of the solution but also strength the coordinate ability of DEA resulting in their anisotropic growth of particles. Some of these particles develop into slim two-dimensional nanosheets. With extending the reaction time, on the one hand, small particles dissappear and large sheet-like particles become bigger 
because of Ostwald ripening process [25-28]. On the other hand, these nanosheets self-assemble and form sphere-like structures to reduce surface energy.

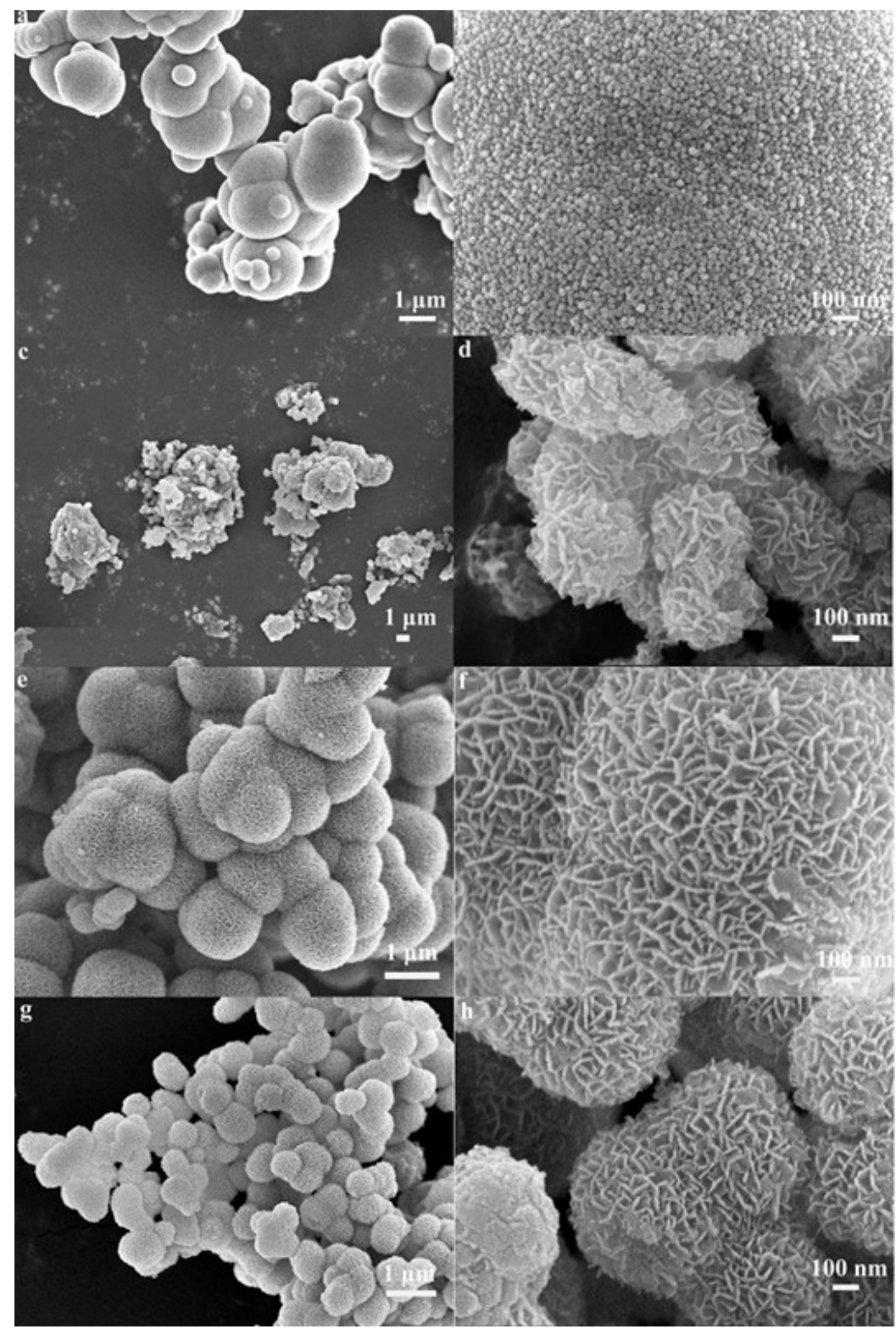

Figure 3 The FE-SEM images of the $\mathrm{TiO}_{2}$ samples synthesized by solvothermal treatment at $200{ }^{\circ} \mathrm{C}$ at different intervals: (a-b) $6 \mathrm{~h},(\mathrm{c}-\mathrm{d}) 12 \mathrm{~h}$, (e-f) $18 \mathrm{~h}$ and (g-h) $24 \mathrm{~h}$

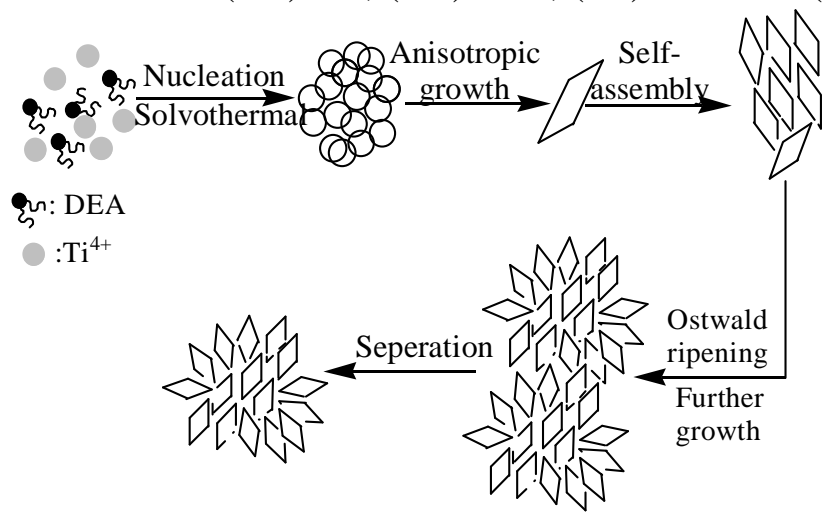

Scheme 1 Schematic illustration of the growth mechanism of $\mathrm{TiO}_{2}$ hiearchical microspheres

Photocatalytic Activity. The photocatalytic activity of $\mathrm{TiO}_{2}$ micriospheres is studied by the photodegradation of methyl orange (MO) under UV light and the results are compared with that of $\mathrm{P} 25$, as shown in Fig. 4. The degradation rate of $\mathrm{MO}$ solutions in the presence of $\mathrm{p} 25, \mathrm{TiO}_{2} \mathrm{samples}$ 
calcinated at $400{ }^{\circ} \mathrm{C}$ are about 20.5 and $91.3 \%$ under UVirradiation for 100 min. The $\mathrm{TiO}_{2}$ sample calcinated at $400{ }^{\circ} \mathrm{C}$ exhibits betterphotocatalytic activity than $\mathrm{p} 25$. This result demonstrates that the morphology of $\mathrm{TiO}_{2}$ microstructure is maintained, and the photocatalytic activity of the sample is signicantly enhanced. When the photodegradation completes, the residue is centrifuged and dried at $60{ }^{\circ} \mathrm{C}$ in air. Then the recycled catalyst is dispersed in a new MO solution and the photodegradation experiment performs again. The $\mathrm{TiO}_{2}$ microsphere catalyst can retain its high activity after three cycles (Fig. 5).

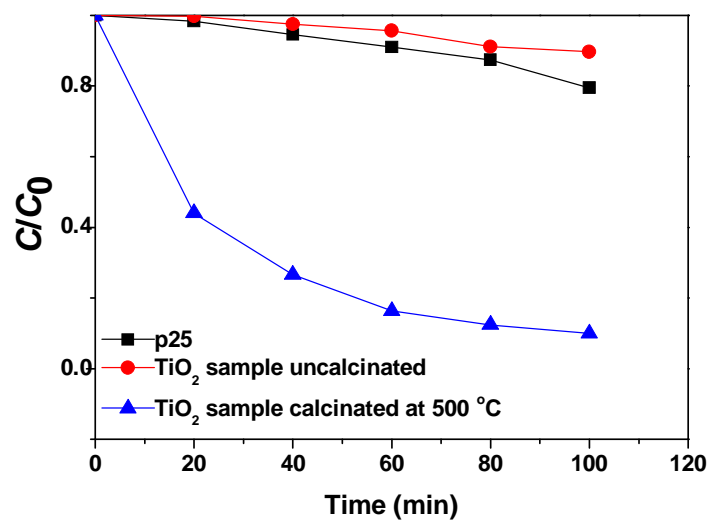

Figure 4 Evolution of MO concentration versus UV irradiation time in the presence of different catalysts

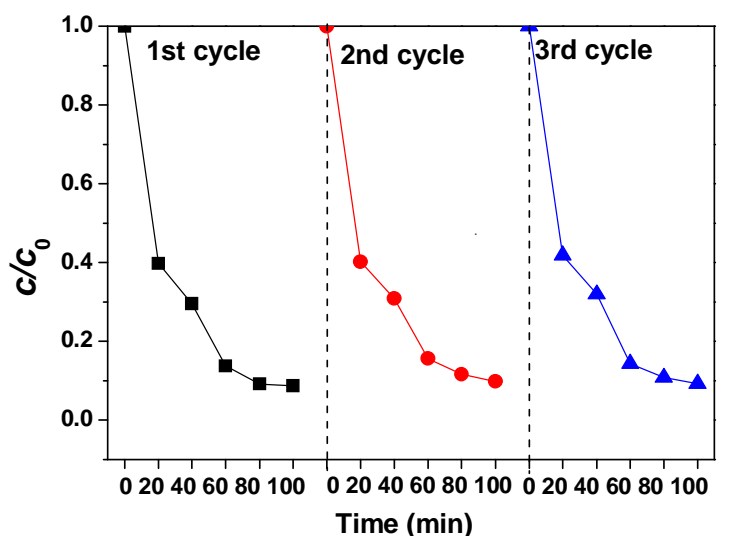

Figure 5 The reusability of the $\mathrm{TiO}_{2}$ sample calcined at $400{ }^{\circ} \mathrm{C}$ as a catalyst for MO photodegradation under UV irradiation

\section{Conclusions}

In summary, three-dimensional hierarchical $\mathrm{TiO}_{2}$ microspheres have been successfully produced via a facile solvothermal approach. The possible formation mechanism is proposed based on time-dependent SEM results. The photocatalytic activity of $\mathrm{TiO}_{2}$ microsphere is enhanced due to the hierarchical structure of microspheres composed of nanosheets. This work may provide a new idea for fabricating $\mathrm{TiO}_{2}$-based photocatalysts with high-performance based on structural design.

\section{Acknowledgements}

The authors would like to thank the National Natural Science Foundation of China (Nos: 51572136 );

Natural Science Foundation of Shandong Provincial (ZR2011BL015).

\section{References}

[1] M.R. Hoffmann, S.T. Martin, W.Y. Choi and D.W. Bahnemann: Chem. Rev. Vol. 95(1995), p. 69

[2] A. Fujishima , T.N. Rao and D.A. Tryk: J. Photochem. Photobiol. C: Photochem. Rev. Vol. 1(2000), p. 1

[3] S.X. Li, J. Chen, F.Y. Zheng, Y.C. Li and F.H. Huang: Nanoscale Vol. 5(2013), p. 12150

[4] Z. Lai , F. Peng, Y. Wang, H. Wang, H. Yu, P. Liu and H. Zhao: J. Mater. Chem. Vol. 22 (2012), p. 23906

[5] D. Chen, F. Huang, Y.B. Cheng and R.A. Caruso: Adv. Mater. Vol. 21(2009), p. 2206

[6] M. Murdoch, G.I.N. Waterhouse, M.A. Nadeem, J.B. Metson, M.A. Keane, R.F. Howe, J. Lorca and H. Idriss: Nat. Chem. Vol. 3(2011), p. 489 
[7] B. Liu and E.S. Aydil: J. Am. Chem. Soc. Vol. 131(2009), p. 3985

[8] S.U.M. Khan, M. Al-Shahry and W.B. Ingler Jr: Science Vol. 297(2002), p. 2243

[9] G.Q. Zhang, H.B. Wu, T. Song, U. Paik and X.W. Lou: Angew. Chem. Int. Ed. Vol. 53 (2014), p. 12590

[10] K. Nakata and A. Fujishima: J. Photochem. Photobiol. C: Photochem. Rev. Vol. 13(2012), p. 169

[11] B. Liu, K. Nakata, M. Sakai, H. Saito, T. Ochiai, T. Murakami, K. Takagi and A. Fujishima: Langmuir Vol. 27(2011), p. 8500

[12] A.K. Alves, F.A. Berutti, F.J. Clemens, T. Graule and C.P. Bergmann: Mater. Res. Bull. Vol. 44(2009), p. 312

[13] T. Shichi and K.-i.Katsumata: Hyomen Gijutsu Vol. 61(2010), p. 30

[14] L.X. Sang, Y.X. Zhao and C. Burda: Chem. Rev. Vol. 114(2014), p. 9283

[15] C. Wang, L. Yin, L. Zhang, Y. Qi, N. Lun and N. Liu: Langmuir Vol. 26(2010), p. 12841

[16] M. Ge, J.W. Li , L. Liu and Z. Zhou: Chem. Res. Vol. 50(2011), p. 6681

[17] Y. Masuda, T. Ohji and K. Kato: Cryst. Growth Des. Vol. 10(2010), p. 913

[18] S.Q. Shang, X.L. Jiao and D.Y. Chen: ACS Appl. Mater. Interfaces Vol. 4(2012), p. 860

[19] Y. Bai, I. Mora-Sero, F.D. Angelis, J. Bisquert and P. Wang: Chem. Rev. Vol. 114(2014), p. 10095

[20] J. Bai and B.X. Zhou: Chem. Rev. Vol. 114(2014), p. 10131

[21] Z.Y. Wang and X.W. Lou: Adv. Mater. Vol. 24(2012), p. 4124

[22] J.P. Wang, Y. Bai, M.Y. Wu , J. Yin and W.F. Zhang: J. Power Sources Vol. 191(2009), p. 614

[23] L. Xiao, M. L. Cao, D.D. Mei, Y.L. Guo, L.F. Yao, D.Y. Qu and B.H. Deng: J. Power Sources Vol. 238(2013), p. 197

[24] S.J. Ding, T.Q. Lin, Y.M. Wang, X.J. Lu and F.Q. Huang: New J. Chem. Vol. 37( 2013), p. 784

[25] Y. Li, Z.Y. Fu and B.L. Su: Adv. Func. Mater. Vol. 22(2012), p. 4634

[26] L.F. Xiao, Y.Q. Zhao , J. Yin and L.Z. Zhang: Chem. Eur. J. Vol. 15(2009), p. 9442

[27] F. Dong, A. Zheng, Y. Sun, M. Fu, B. Jiang, W.-K. Ho, S. C. Lee and Z. Wu: CrystEngComm Vol. 14(2012), p. 3534

[28] C.C. Yec and H.C. Zeng: J. Mater. Chem. A Vol. 2 (2014), p. 4843 